\title{
A Fuzzy Logic Adaptive Image Compression Level using Cross-Layering in Wireless Multimedia Sensor Networks
}

\author{
Mohammed Ameen A. Abdo \\ College of Engineering, Alzaiem \\ Alazhari University, Khartoum, \\ Sudan \\ College of Engineering, Azal \\ University of Human Development, \\ Sana'a, Yemen
}

\author{
Ala Eldin Abdallah Awouda \\ University of Bisha College of \\ Engineering \\ Bisha, Saudi Arabia \\ Sudan University of Science and \\ Technology \\ College of Engineering \\ Khartoum, Sudan
}

\author{
Yousif Elfatih Yousif \\ College of Engineering, Alzaiem \\ Alazhari University, Khartoum, \\ Sudan
}

\begin{abstract}
The growing interest in Wireless Sensor Networks (WSNs) with the rapid growth in micro-electronics technology has made it possible to deliver multimedia content over Wireless Multimedia Sensor Networks (WMSNs). There are several main peculiarities that make the delivery of multimedia content over WMSN challenging. Most of these are due to the processing, timing, and other quality of service requirements. Furthermore, WMSNs are susceptible to rapid degradation since they deal with large amount of data that require processing and transmission power. In this paper, a crosslayer design approach is proposed to overcome such challenges. In the proposed model, the concept of crosslayering and fuzzy logic has been exploited to monitor the network conditions and control the amount of the multimedia data in order to utilize the available resources efficiently and improve the applications Quality of Service (QoS). The simulation results have shown better resource utilization, stability, and fairness in quality metrics consideration. The proposed model has shown to be efficient compared to the conventional scheme in terms of bandwidth utilization, power consumption, delay, loss, and images quality.
\end{abstract}

Keywords

Image compression, WMSN, Cross-layer, Fuzzy

\section{INTRODUCTION}

WSNs are a set of devices equipped with the necessary equipment to carry out their mission in the region of interest. Network lifetime, data quality, and transmission speed are important factors in some networking applications such as multimedia and real-time applications. WSNs, which require distributing devices equipped with limited resources on an environment in isolation from supplement sources, are vulnerable to quick deterioration in performance and reduction of lifetime rapidly due to the consumption of the limited resources. Many studies and research concentrate on reducing the resource consumption and improving the network performance as much as possible [1]. Recent studies have focused on dealing with more than one layer in the network stack and at the same time trying to get the best possible performance of the network according to the requirements of the application [2].

Dealing with more than one layer at the same time is known as the principle of cross layering. In this study, this concept is applied along with AI Artificial Intelligence to control the behavior of the network periodically in order to ensure the best utilization of the available resources, and hence to ensure the best possible QoS. In this research, multimedia application in WSNs has been focused. An intelligent controller is used to control the behavior of the network layers in an integrated manner, so that all the layers will be aware of what is going on in the other layers. Based on this, each layer can send control messages to the relevant layers to modify their behavior, either temporarily or permanently, in order to recover the stability of the network and reduce the consumption of its resources.

The architecture of the WSNs consists of five layers: application, routing, radio, Media Access Control (MAC), and physical [3]. In this study, all the layers is focused except the routing layer as it contains a set of protocols dedicated to deal with the conditions and requirements of different applications, such as Ad hoc On-Demand Distance Vector (AODV), LowEnergy Adaptive Clustering Hierarchy (LEACH), Greedy Perimeter Stateless Routing (GPSR), multipath, and bypass routing protocols. Accordingly, this study can be applied on different routing protocols, and then the appropriate protocol that guarantees the best performance can be chosen. In order to get realistic results, the application layer will deal with multimedia sensors distributed in the environment with virtual targets, mobile and fixed, and the line of sight of these sensors can be adjusted at certain angles. Furthermore, the application layer will deal with real images, and simulate the processing, compression, and transmission of the images over the network with the ability to test the quality of the transmitted images when passing through any node in the network. The application layer also will take into account the energy consumed during capturing, processing, compression, and transmission of the images over the network. Consequently, the simulation results can be used to improve the performance of the network by modifying and controlling the normal behavior of the layers to get the best possible QoS.

\section{FUZZY MODELS}

A Fuzzy system mainly consists of three units: fuzzifier, inference engine, and defuzzifier [4]. Figure 1 shows the components of the traditional fuzzy system model. Input fuzzification is the first step in creating fuzzy model of a system. In this step, fuzzy membership functions are applied to the system inputs to specify the degree of membership for the input crisp values. This means that assigning a group memberships and membership values to the system inputs. The second step is to take the fuzzified inputs and apply them to the system fuzzy rules. Fuzzy rules are series of if-then statements, where the "if" side is called the condition, and the 
"then side" is the conclusion. The last step is the defuzzification of the fuzzy output sets by which all the actions are companied and converted into a single non-fuzzy value to represent the output of the system. There are many of defuzzification methods such as centroid method, Maxmembership method, Weighted average method, and Middle of maxima each method has its pros and cons regarding complexity, accuracy, and speed [5].

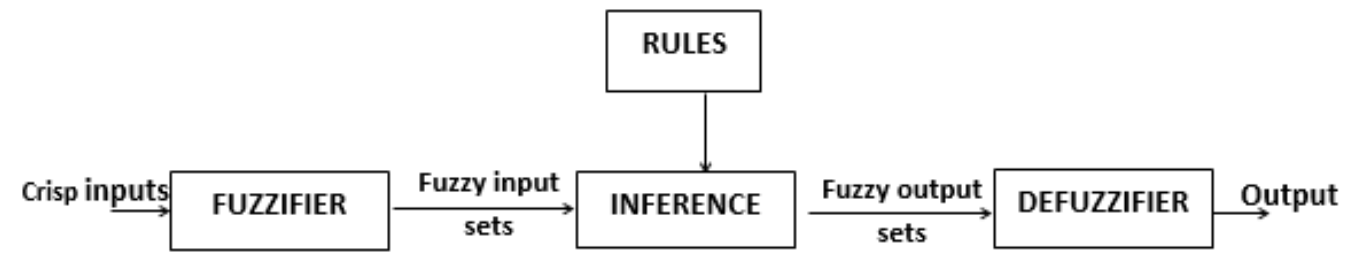

Figure 1: Block Diagram of a Fuzzy Logic System

\section{THE PROPOSED FRAMEWORK}

Figure 2 shows the proposed cross-layer system architecture. As shown, the proposed model is designed according to the bottom up architecture, in which the upper layers change their traditional behaviors in order to adapt to the changes occurred in the lower layers. The layers that are involved in the system architecture are the physical layer, the network layer, and the application layer.

The left side of the figure shows the stack layer architecture for WSNs, while the right side shows the corresponding layer parameters used as system inputs. Also the right side shows the system output which represent the system feedback to the application layer to adjust the image compression level.
The adaptive model sends frequent control messages to the application layers of the source nodes to change their behaviors according to the information obtained from the other layers.

The system here deals with the average power consumed by all the sources, not for each source node independently, to prevent the sources from sending their own power independently from each other which may make variations in the received images and discrepancy in compression level at the receiver side. The system also aims to make smooth transition in images compression level to give the receiver the chance to receive images organized as groups according to their compression level. So, the adaptive model here sends uniform control messages to all of the sources.

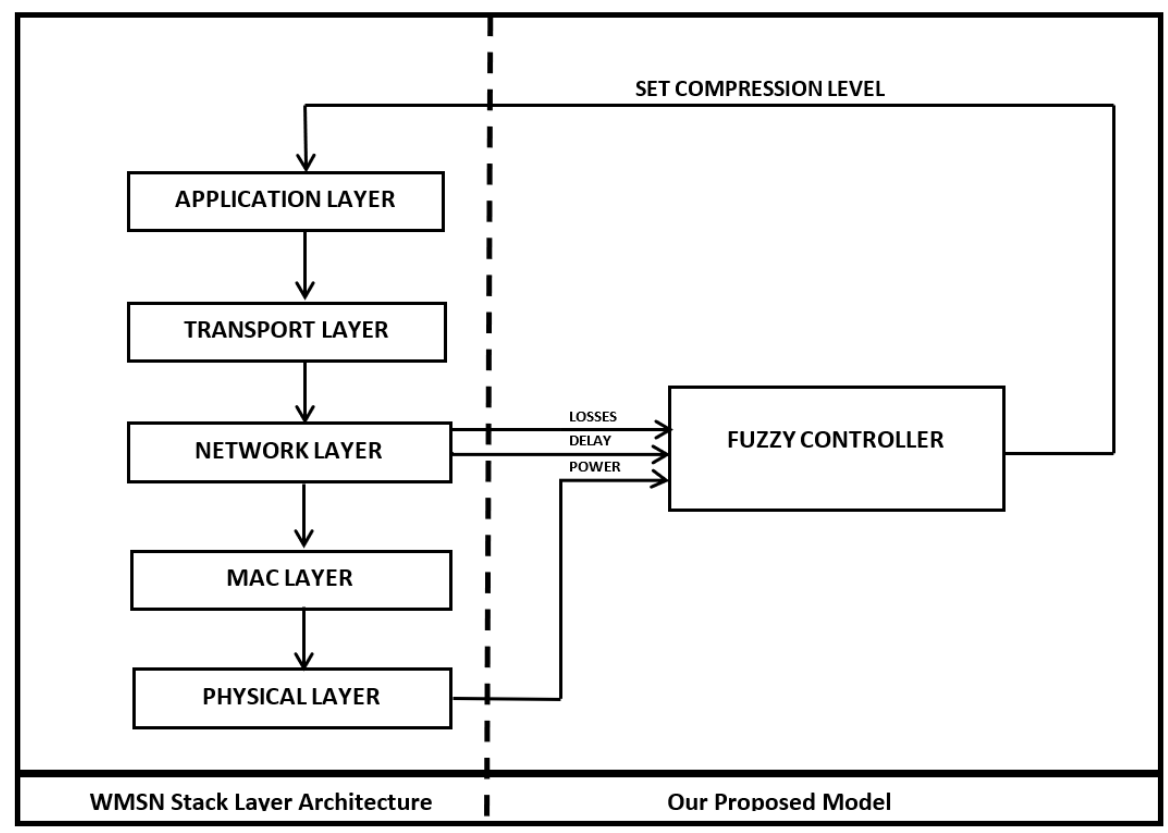

Figure 2: The Proposed Framework

In proposed model the previous three ideas (cross-layering, adaptation, and fuzzy logic) is combined to enhance the QoS and to meet the requirements of the applications that the network dedicated for. Merging those ideas will defiantly support the maximum efficiency.

Furthermore, proposed model used an AI controller. Precisely, fuzzy logic is used to build our controller while other approaches use other techniques. [6], [7] and [8] used neural network technique to improve power and images quality in WSNs. [9] and [10] used the genetic algorithm to improve the coverage and lifetime. Also [11] used the hill climbing technique to improve the coverage in the mobile sensor networks.

Therefore, as mentioned previously, fuzzy logic technique has been elected in proposed model to improve the processing time and decrease processing overhead in the nodes. In addition, fuzzy logic improves the flexibility to reconstruct the behavior of the controller to meet the requirements of other network applications if needed. 


\subsection{Network Simulator (Castalia 3.2)}

OMNeT++ is one of the most popular WSN simulators [12]. Castalia is an OMNeT++ package dedicated for WSN projects simulation [13]. NS3 is another popular simulator which is considered the strongest competitor of OMNeT++. Recently, Videos model package has been adding to Castalia which is dedicated to handle multimedia WSNs WMSN which is another wireless sensor networking approach. Castalia proved its efficiency regarding dealing with real multimedia content such as images and videos to be the first simulator able to cope with real multimedia files, taking into account all the details while simulating the transmission of multimedia contents.

The internal structure of the node is shown in Figure 3. The bold arrows show the message passage between layers protocols stack while the dashed arrows represent the function call so all of the layers has the capability to call the function of the source manager to deal with power which is managed inside this model, all the layers can communicated with each other directly or indirectly using the parent model or layer as an interface [14].

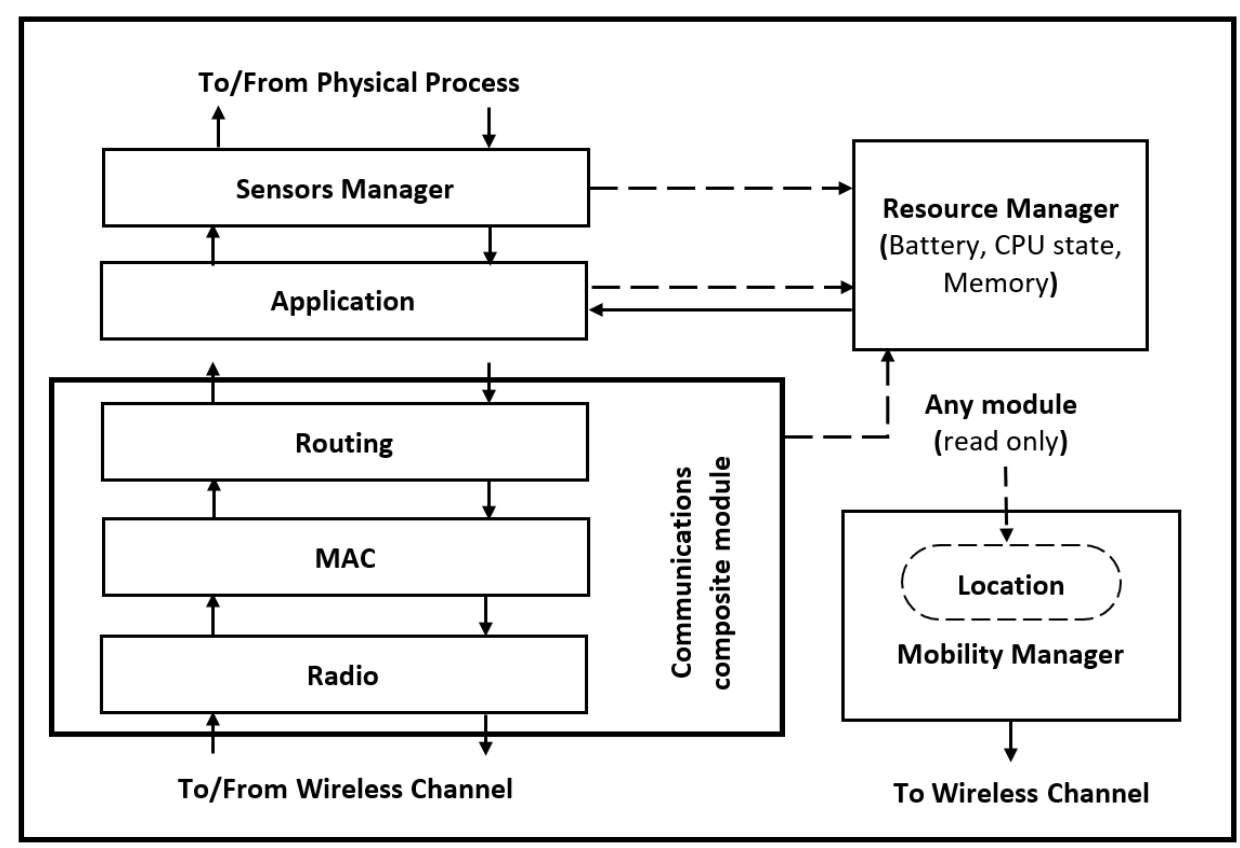

Figure 3: Castalia Node's Composite Module

\subsection{Power Simulator (xeemu)}

XEEMU is a power simulator dedicated for the Intel(c) XScale-based systems. XEEMU is also a configurable runtime simulator with very high accuracy, the average error is $3.0 \%$ for runtime and $1.6 \%$ for the estimation of CPU energy consumption. This simulator can be used together with another application to compute the delay and power consumed during the operation of this application [15].

\subsection{Utility Applications (CJPEG)}

CJPEG is stand for Compress an image file to a JPEG file. This application was designed to implement the compression and decompression operations for JPEG images. JPEG is lossy, which means that the output image is not necessarily to be identical to the input image.
This application is used for compress and resizing images before being sent by source nodes, also this application is used together with Xeemu simulator to provide us with accurate information about the power consumed and processing delay during compression and resizing operations [16].

\subsection{The Proposed Fuzzy Inference Model (FIM)}

The proposed FIM is designed based on the Mamdani model. The system was conceived in the light of many experiments applied to different scenarios with different conditions. The proposed FIM consists of three inputs as shown in Figure 4 These inputs are the average remaining power in the network, the average network delay, and the average loss rate.

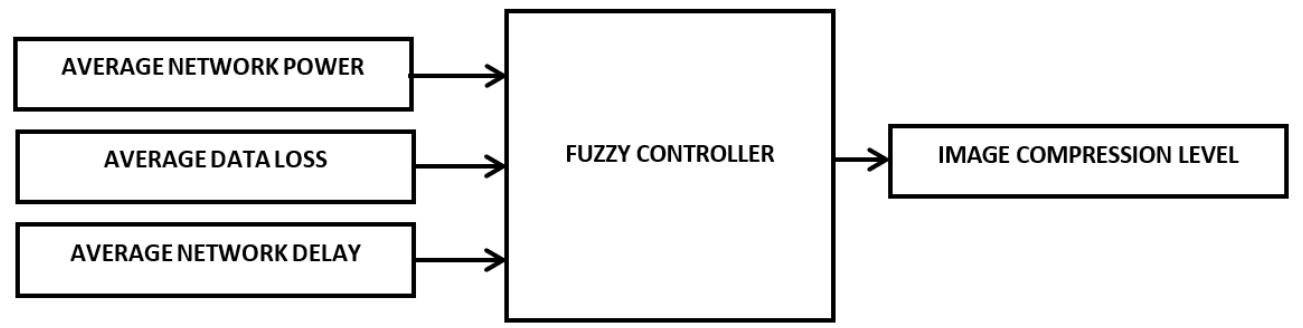

Figure 4: Fuzzy Model Architecture

\section{RESULT AND DISCUSSION}

During obtaining results, the adaptive model has been implemented on different scenarios taking into account the effect of adapting images quality on the other quality factors. 
So, this section the effect of adapting image quality will be studied and will be concluded with general discussion of the results obtained from one of the most congested scenarios to show the efficiency of the proposed model in the most difficult cases.

This experiment was aimed at stimulating the worst conditions that the networks may face. The simulation parameters used in the experiments are shown in Table 1.

Table 1: Simulation Parameters

\begin{tabular}{|c|c|}
\hline Parameters & Values \\
\hline Network size & $200 \times 200 \mathrm{~m} 2$ \\
\hline Number of nodes & 50 nodes \\
\hline Packet size & 256 bytes \\
\hline Images transmission & $10-70 \mathrm{sec}$ \\
\hline Communication range & $30 \mathrm{~m}$ \\
\hline Routing protocol & GPSR \\
\hline MAC protocol & TMAC \\
\hline Initial energy & $50 \mathrm{~J}$ \\
\hline Simulation time & $400 \mathrm{c}$ \\
\hline
\end{tabular}

\subsection{Analysis}

From previous experiment, a clear gradient can be seen in the QoS factors. Of course, the lower the amount of transmitted data, the better the performance of the network, but this procedure may spark many trade-off problems, reducing the number of transmitted data will reduce the quality of the received data. In WMSNs, sending a large amount of data can turn situation from bad to worse specially if all of the sources send their data over the network at the same time. In this case, the network may suffer from high loss rate, which is unacceptable in sensitive applications such as multimedia applications, also receiving distorted or unreadable data will waste network resources.

Table 2: The Percentages and Average Compression Level of the Transmitted Images Using the Adaptive and CSE Model

\begin{tabular}{|c|c|c|}
\hline $\begin{array}{c}\text { Compression level } \\
\text { percentage }\end{array}$ & CSE Model & $\begin{array}{c}\text { Proposed } \\
\text { No of }\end{array}$ \\
\hline & No of Transmitted & 2 \\
\hline 100 & 2 & \\
\hline 79 & 1 & \\
\hline 68 & 1 & \\
\hline 66 & 1 & \\
\hline 59 & 1 & \\
\hline 57 & 1 & \\
\hline 56 & 5 & \\
\hline
\end{tabular}

\begin{tabular}{|c|c|c|}
\hline 55 & 5 & \\
\hline 54 & 3 & \\
\hline 53 & 12 & \\
\hline 52 & 11 & \\
\hline 51 & 7 & \\
\hline 48 & 12 & \\
\hline 47 & 10 & \\
\hline 45 & 11 & \\
\hline 40 & & 21 \\
\hline 38 & & 5 \\
\hline 36 & & 10 \\
\hline 34 & & 4 \\
\hline 32 & & 72 \\
\hline 24 & 1 & \\
\hline $\begin{array}{c}\text { No of Transmitted } \\
\text { Images }\end{array}$ & 88 & 114 \\
\hline $\begin{array}{c}\text { Average } \\
\text { Compression Level }\end{array}$ & $52 \%$ & $51 \%$ \\
\hline
\end{tabular}

The lower compression level factor at the sender side is not an indication of bad quality at the receiver side. In contrast, sending images with high quality with bad network conditions makes them violated to more quality deterioration. So, the proposed model chooses the suitable quality compression level according to the network conditions to ensure the best quality at the receiving side.

Images compression level has direct effect on the amount of data being sent which also effect the power required to process and send these data as shown in Figure 5. So, the proposed model as shown in Table 2 spends lower power and processing time during compression in the application layer and also in the radio layer during transmission.

From another hand, the proposed model proves its efficiency by sending more images compared to CSE. As shown in Table 2, the proposed model sent 114 images compared to 88 images sent by the CSE model.

Also the widening and narrowing of the gap between the proposed and CSE model is noticed according to the instantaneous conditions of the network. Also, who does the adaptive model return to the aggressive mode regarding to power consumption over the time whenever network situations start getting worse can be noticed. As shown in Figure 5, the end of power timeline for the proposed model is quite less than CSE model despite the proposed model was able to send more images as mentioned previously in Table 2 which prove the efficiency of proposed model regarding power consumption. 


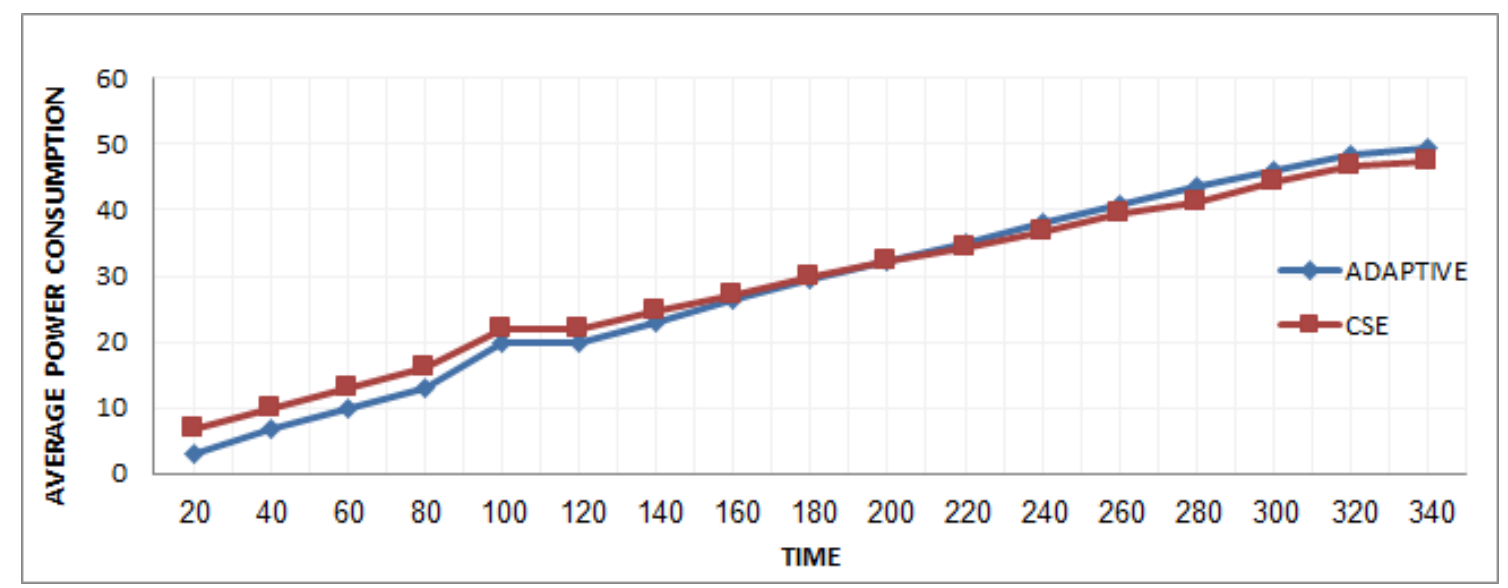

Figure 5: Power Consumption

Balancing the amount of power consumption in a network may also led to better utilization of network bandwidth, So the improvement of the lifetime of the source nodes or even the relay nodes may give the network a chance for sending more images. As what has been mentioned previously, the proposed model keeps track of the power consumption of the network periodically. This policy can improve balance the power consumption in the network and may give it the chance for sending more images because the death of a source node or sensitive relay node may hinder the network from sending more images. So, the source node may prefer to send images with lower quality instead of sending images with higher or full quality if the source nodes power is getting worse, therefor significant improvement can be noticed regarding to the number of sent images as shown in Figure 5.

Figure 6 shows the number of alive nodes over time for the proposed and CSE models. The relation between the average power consumption and the number of alive nodes can be noticed. As shown in the figure, the proposed model and CSE are almost the same at the beginning of the simulation time, and then that the number of nodes start decreasing as a result of lost their power can be noticed, specially the critical nodes at the model of data path to the sink as result of overwhelming these nodes by sending them more traffic specially when sending images with higher compression factor.

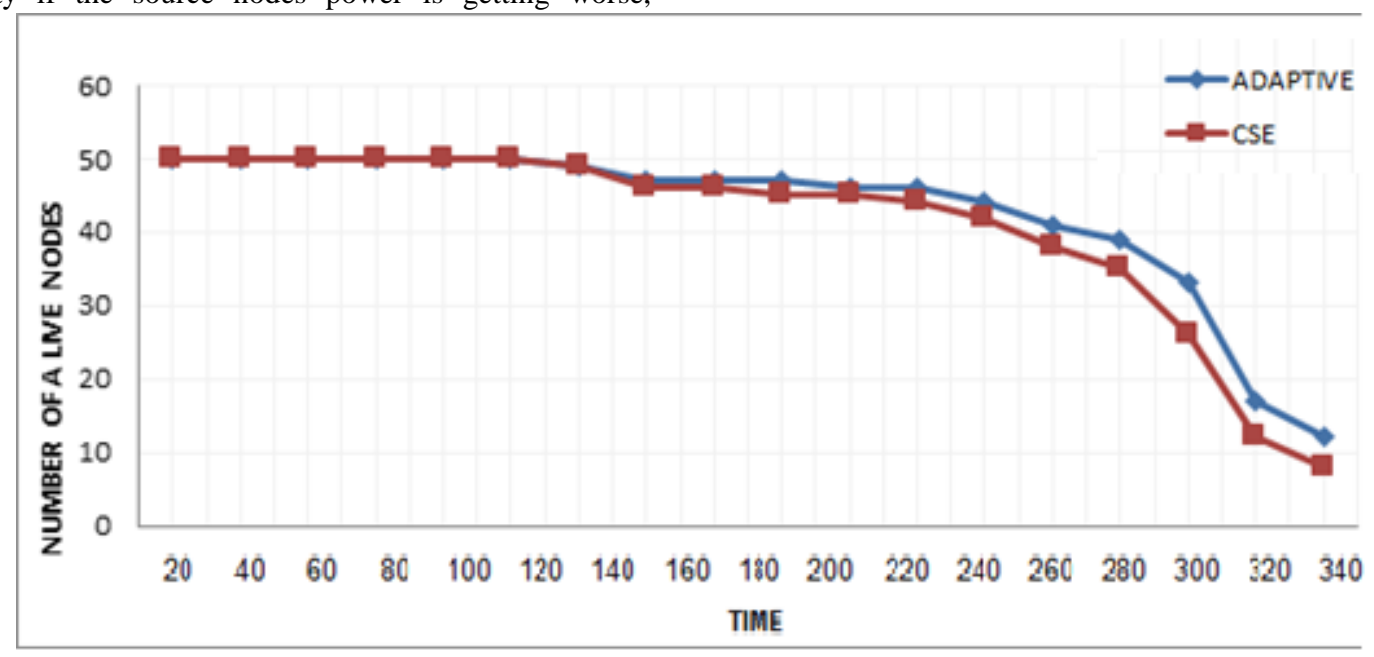

Figure 6: Number of Alive Nodes

Flooding the network with data, especially when more than one source sends their data over the network, may overwhelm the network causing data congestion and bottleneck in the nearest node to the sink. This also may have led to speed up the lifetime of the relay nodes making a gap or alternative long path to the sink. All of these may affect the network data delivery ratio by increasing the data loss rate. Figure 10 shows the timeline of the loss ratio for the proposed and CSE models. As shown in Figure 7, the proposed model achieve lower percentage of data loss compare to CSE can be noticed. The figure shows higher loss percent at the beginning of run time, so the proposed model immediately starts being more aggressive by sending images with lower quality to cope with network congestion and decrease data loss ratio. As shown in Table 3, the proposed model can be noticed that has the ability to send images with average compression level equal to $51 \%$ compared to $52 \%$ in CSE. This decreasing helps the network to decrease the congestion and loss ratio which also can directly effects the quality of the images at the receiver side. The lower loss ratio is the better quality. The small value of average compression level for large number of images means large amount of packets compare to the value of average compression factor for small number of images. This means that the network with the proposed model was able to send more data as shown in Table 3. 


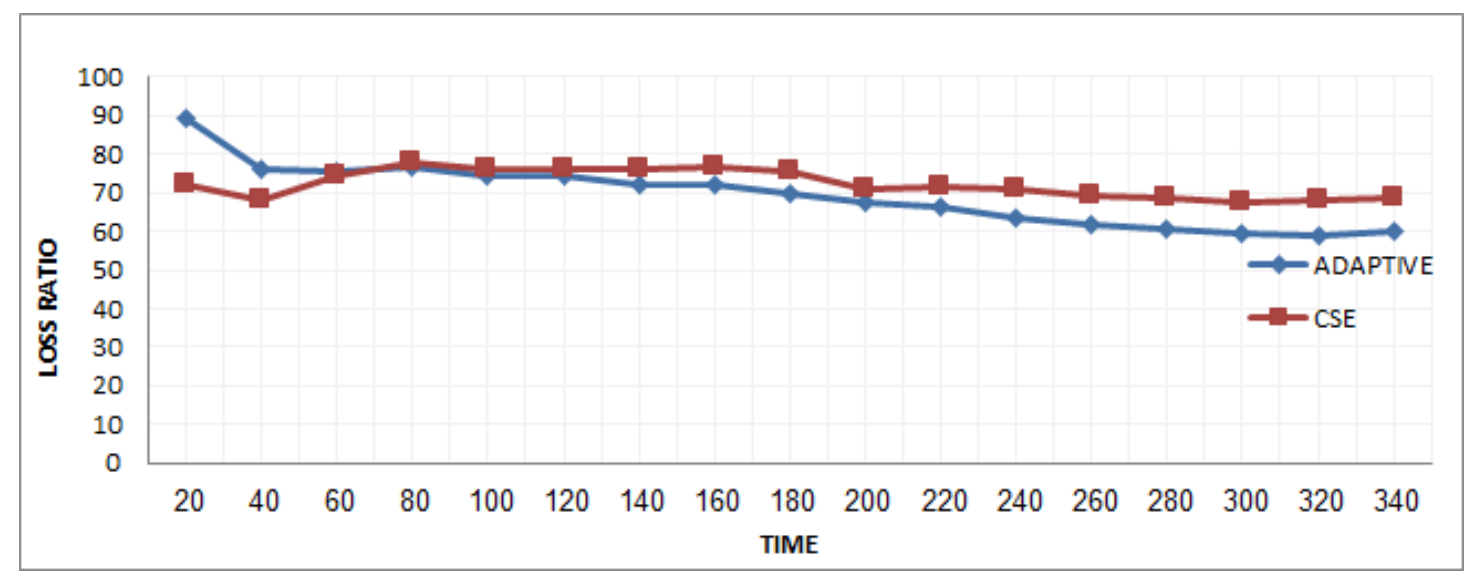

Figure 7: Loss Ratio

As mentioned previously, flooding network with data may led to network bottleneck and data congestion causing more delay. The proposed model was designed to cope with the requirements of the variations of multimedia applications such as images transmission or video streaming applications over WSNs. Also it was designed to cope with the requirements of these applications over wide geographical area. Real time applications over WSNs have their strict requirements regarding to time constrains which can be tolerant up to specific delay threshold. The average delay of the previous scenarios was in the almost low which is eligible to meet the requirements of these applications this because of the small and central based sink position networks. So good behavior of the proposed and CSE models can be noticed. Figure 8 shows the average network delay when using the proposed and CSE models. The efficiency of the proposed model from the timeline can be noticed. The proposed model is returned to decrease the network congestion by decreasing network traffic by adapting the compression level for the images before being sent.

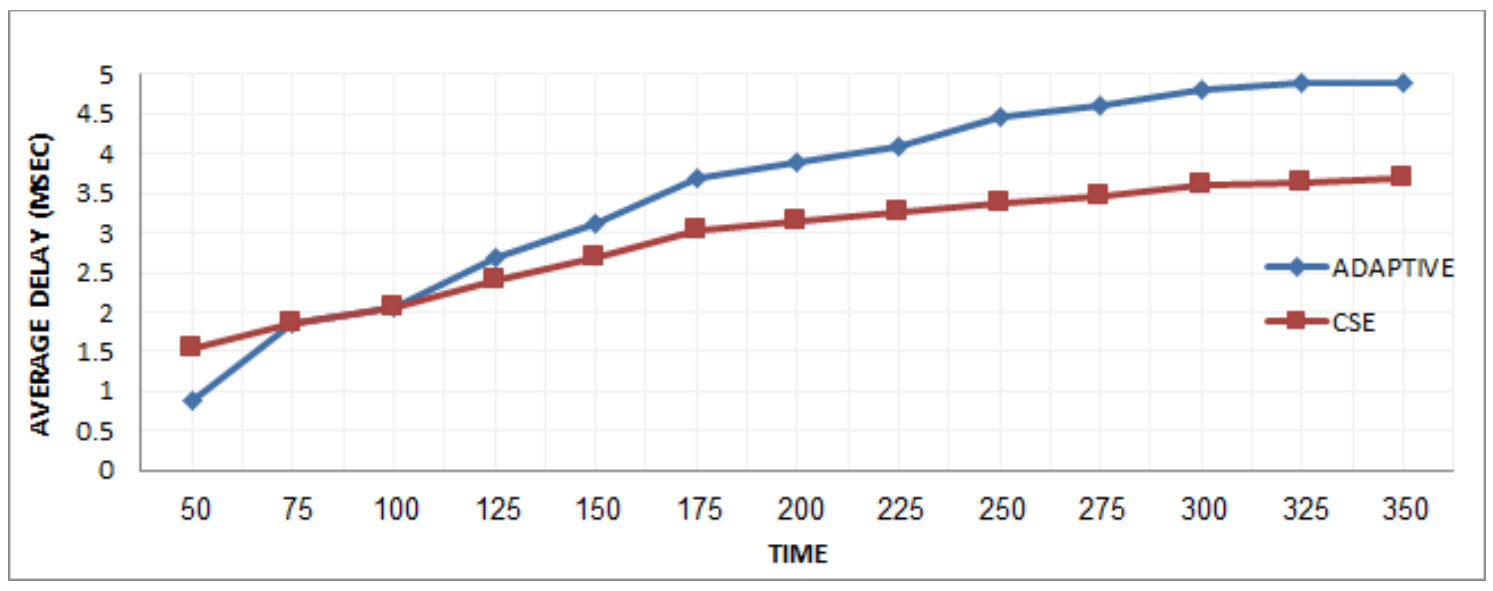

Figure 8: Delay

In the previous sections, the behavior of the proposed model and CSE regarding power consumption, data loss, and delay metrics will be discussed. Now the quality of the received images using the previous algorithms will be discussed.

Figure 9 shows the PSNR of the received images for the proposed model and CSE. The figure proves the efficiency of the proposed model over the CSE. The deregulation of the quality of the images when using the CSE specially at the beginning of the simulation can be noticed as a result of sending images with high compression level without estimating the networks conditions in contrast to the proposed model which start with reasonable compression level according to the network conditions taking into account the different quality factors that affect the quality of images such as loos and delay. 


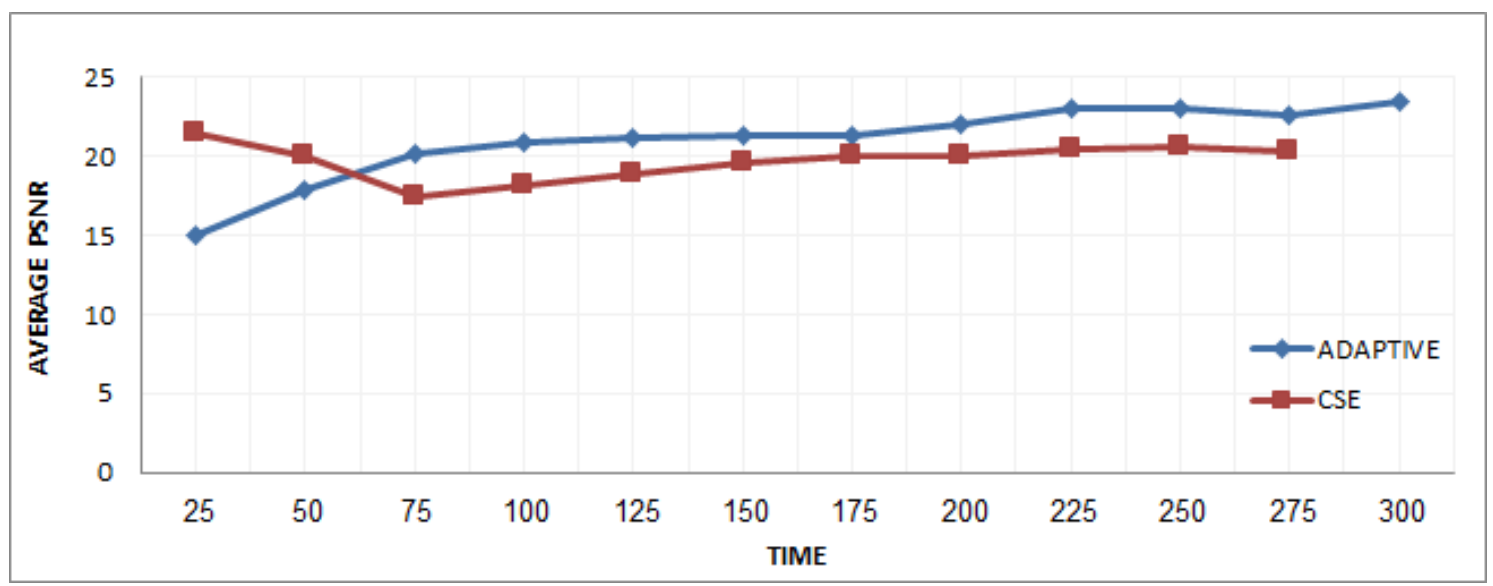

Figure 9: Average PSNR

\section{CONCLUSION}

In this work, a fuzzy logic-based cross-layer design model has been proposed to improve the QoS requirements in WMSNs. The proposed model applies compression adaptation to provide smooth transition in the amount of generated traffic according to the network conditions in order to improve the overall network performance and resource utilization while maintaining the best possible visual quality at the receiver side.

In order to evaluate the system performance, transmission process of real images is simulated with taking into account different network scenarios and used power simulator to consider the power consumed in image processing at the application layer. The simulation results showed the efficiency of the proposed system in terms of resource utilization, fairness between the quality metrics, stability, and eventually improving the quality of the received data. The proposed model has proved its efficiency compared to the other adaptive method in terms of power consumption, delay and loss.

\section{REFERENCES}

[1] M. H. Alsharif, S. Kim, and N. Kuruoğlu, "Energy harvesting techniques for wireless sensor networks/radiofrequency identification: A review," Symmetry, vol. 11, no. 7, pp. 865-865, 2019.

[2] L. Li, Y. Liu, J. Wang, T. Wu, and P. Zhou, "Partially observed cross-layer optimization for vehicular communications," vol. 31, no. 1, p. e3398, 2018.

[3] V. J. I. J. o. A. R. i. C. E. Jindal and Technology, "History and architecture of wireless sensor networks for ubiquitous computing," vol. 7, no. 2, pp. 214-217, 2018.

[4] A. K. Dwivedi and A. Sharma, "FEECA: Fuzzy based Energy Efficient Clustering Approach in Wireless Sensor Network," EAI Endorsed Transactions on Scalable Information Systems, vol. 7, no. 27, 2020.

[5] I. P. Ktistakis, G. Goodman, and C. Shimizu, "Methods for optimizing fuzzy inference systems," in Advances in Data Science: Methodologies and Applications: Springer, 2020, pp. 97-116-97-116.

[6] Y. Zur and A. Adler, "Deep Learning of Compressed Sensing Operators with Structural Similarity Loss," arXiv preprint arXiv:1906.10411, 2019.

[7] L. B. Bhajantri, "A Comprehensive Survey on Data
Aggregation in Wireless Sensor Networks," 2018.

[8] R. Song, Q. Wei, and W. Xiao, "ADP-based optimal sensor scheduling for target tracking in energy harvesting wireless sensor networks," Neural Computing and Applications, vol. 27, no. 6, pp. 1543-1551-1543-1551, 2016.

[9] S. Q. Mahdi, S. K. Gharghan, and M. A. Hasan, "FPGABased neural network for accurate distance estimation of elderly falls using WSN in an indoor environment," Measurement, vol. 167, pp. 108276-108276, 2021.

[10] Z.-J. Wang, Z.-H. Zhan, and J. Zhang, "Solving the energy efficient coverage problem in wireless sensor networks: A distributed genetic algorithm approach with hierarchical fitness evaluation," Energies, vol. 11, no. 12, pp. 3526-3526, 2018.

[11] T. Khampeerpat and C. Jaikaeo, "Mobile sensor relocation for nonuniform and dynamic coverage requirements," IEICE TRANSACTIONS on Information and Systems, vol. 100, no. 3, pp. 520-530-520-530, 2017.

[12] D. Sahin and H. M. Ammari, "Programming Languages, Network Simulators, and Tools," in The Art of Wireless Sensor Networks: Springer, 2014, pp. 739-788-739-788.

[13] M. Bakni, L. M. M. Chacón, Y. Cardinale, G. Terrasson, and O. Curea, "WSN simulators evaluation: an approach focusing on energy awareness," arXiv preprint arXiv:2002.06246, 2020.

[14] K. A. Ngo, T. T. Huynh, and D. T. Huynh, "Simulation wireless sensor networks in castalia," in Proceedings of the 2018 International Conference on Intelligent Information Technology, pp. 39-44-39-44.

[15] Z. Herczeg, Á. Kiss, D. Schmidt, N. Wehn, and T. Gyimóthy, "XEEMU: An improved XScale power simulator," in International Workshop on Power And Timing Modeling, Optimization and Simulation, pp. 300-309-300-309.

[16] S. Sohoni, R. Min, Z. Xu, and Y. Hu, "A study of memory system performance of multimedia applications," ACM SIGMETRICS Performance Evaluation Review, vol. 29, no. 1, pp. 206-215-206-215, 2001. 